\title{
Additives for Ambient 3D Printing with Visible Light
}

\author{
Dowon Ahn ${ }^{\S}$, Lynn M. Stevens ${ }^{\S}$, Kevin Zhou, and Zachariah A. Page* \\ Department of Chemistry, The University of Texas at Austin, 105 East 24th Street, Stop A5300, \\ Austin, Texas 78712, United States. \\ ${ }^{\S}$ Equal contributors \\ *E-mail: zpage@utexas.edu
}

Keywords: 3D printing, digital light processing, visible light, photochemistry, photocuring, polymers

With 3D printing we desire to be "limited only by our imagination", and although remarkable advancements have been made in recent years the scope of printable materials remains narrow compared to other forms of manufacturing. Light-driven polymerization methods for 3D printing are particularly attractive due to unparalleled speed and resolution, yet the reliance on high energy UV/violet light in contemporary processes limits the number of compatible materials due to pervasive absorption, scattering, and degradation at these short wavelengths. Such issues can be addressed with visible light photopolymerizations. However, these lowerenergy methods often suffer from slow reaction times and sensitivity to oxygen, precluding their utility in 3D printing processes that require rapid hardening (curing) to maximize build speed and resolution. Herein, multifunctional thiols (1-10 wt $\%)$ are identified as simple additives to enable rapid ( $\sim 10 \mathrm{~s}$ /layer) high resolution $(<50 \mu \mathrm{m})$ visible light (red, $\sim 620 \mathrm{~nm}) 3 \mathrm{D}$ printing under ambient (atmospheric $\mathrm{O}_{2}$ ) conditions that rival modern UV/violet-based technology. The present process is universal, providing access to commercially relevant acrylic resins with a range of disparate mechanical responses from strong and stiff to soft and extensible. Pushing forward, the insight presented within this study will inform the development of next generation 3D printing materials, such as multicomponent hydrogels and composites.

\section{Introduction}

Additive manufacturing is a rapidly expanding field that enables the production of complex 3D objects with unmatched speed and precision. Unlike traditional "subtractive" manufacturing processes, which require excessive time, energy, material, and equipment (for milling, carving, 
and machining), 3D printing operates in a simple "additive" fashion to create objects via successive growth. ${ }^{[1-3]}$ However, progress from prototyping to industrial manufacturing necessitates continued research efforts in the fundamental science and engineering of 3D printing to simultaneously decrease production time, ${ }^{[4,5]}$ broaden material scope, ${ }^{[6,7]}$ demonstrate energy efficient scalability, ${ }^{[8]}$ and enhance resolution, ${ }^{[9,10]}$ all with facile userfriendly operation.

Numerous additive methods that bring materials together with spatial control have been demonstrated, from deposition and heating to light exposure. The utility of light is particularly attractive given the ability to create micron-sized features at low cost using inexpensive existing projection-based digital light processing (DLP) and light emitting diode liquid crystal display (LED/LCD) technologies. Both DLP and LED/LCD methods use vat photopolymerization 3D printing, which employs light to convert liquid resins into solid objects. Although conventional light-based 3D printing relies on high energy ultraviolet (UV) light to achieve rapid polymerization rates and correspondingly short build times ( seconds/layer), visible light is emerging as an attractive alternative. ${ }^{[11-13]}$ Relative to UV light, visible light is naturally abundant, less expensive, and benign (generally reduced absorption and scattering), which gives it the potential to advance 3D printing of polymeric materials by enabling economical preparation of, for example, cell-laden hydrogels, ${ }^{[14]}$ opaque composites, ${ }^{[15]}$ or multimaterial structures (via wavelength selective reactions). ${ }^{[7,16-19]}$

Recently, we demonstrated that a three component photosystem comprising a visible light absorbing photoredox catalyst (PRC) with donor (diphenyliodonium) and acceptor (triphenyl (n-butyl) borate) co-initiators could facilitate photocuring of acrylate-based resins on timescales ( seconds) that were competitive with commercial UV light systems (Figure 1). ${ }^{[11]}$ However, the difference in mechanism undergone by UV photoinitiators (Type I) and visible PRCs (Type II) to generate reactive radicals results in greater oxygen sensitivity for the latter. Thus, a major limitation of highly reactive visible light curable resins for $3 \mathrm{D}$ printing is the need for oxygen 
removal, both from the resin and surrounding atmosphere during printing. To briefly explain the disparity in oxygen sensitivity, UV photoinitiators generate free radicals upon rapid homolytic scission from singlet photoexcited states (Type I mechanism), while efficient visible PRCs often rely on triplet photoexcited states to minimize the impact of rate-limiting electron transfer to a co-initiator for radical formation (Type II mechanism). ${ }^{[20-22]}$ Given that molecular oxygen resides in a triplet ground state, it rapidly reacts with PRCs in their triplet-excited states, which results in a delayed onset of curing, called the oxygen inhibition period. ${ }^{[23]}$ Furthermore, oxygen will quench propagating carbon centered radicals to form more stable peroxy radicals that do not reinitiate acrylate polymerizations. Thus, oxygen limits curing speed and monomerto-polymer conversion, which necessitates its removal. ${ }^{[24,25]}$

Oxygen inhibition time has previously been decreased for photocurable coatings by increasing photo-catalyst/-initiator concentrations ( $\geq 5 \mathrm{wt} \%)$, which causes a rapid reaction with ambient oxygen. ${ }^{[25]}$ However, increased loadings bear a number of limitations, including associated costs, requirements for resin solubility, reduced penetration depths due to optical attenuation, increased toxicity, and plasticization (softening) of cured parts. Alternatively, several physical strategies for eliminating oxygen inhibition have been developed for industrial coatings, such as photocuring under inert gas (e.g., $\mathrm{N}_{2}$ or Ar), introducing physical barriers (e.g., in-laminate), employing resins with low oxygen solubility, and increasing irradiation intensity. ${ }^{[25]}$ Despite success in coatings, these approaches limit the general applicability of visible light $3 \mathrm{D}$ printing by requiring specialized and costly equipment. Chemical deoxygenation (e.g., oxygen scavengers) presents a low-cost and facile substitute. Apart from decreasing or removing the oxygen inhibition period, ideal chemical additives should not slow the photopolymerization, generate toxic byproducts that can leach out of the final print, nor alter thermomechanical properties of the printed object.

Inspired by oxygen insensitive thiol-ene photopolymerizations, ${ }^{[26,27]}$ we examined the use of thiols as additives to enable ambient 3D printing of acrylic resins with red light $(\sim 620 \mathrm{~nm})$. 
In contrast to pure thiol-ene resins, acrylic resins have a greater commercial presence due to their more tenable odor, improved storage stability, and wider materials scope. Described herein is the unprecedented impact of thiol additives to simultaneously preclude oxygen inhibition and enhance photopolymerization rates under ambient conditions for visible light photocuring. The process is shown to be universal, enabling rapid high-resolution visible light $3 \mathrm{D}$ printing for an array of acrylic resins. To emphasize industrial relevance, commercial urethane acrylate resins from Dymax ${ }^{\circledR}$ serve as a representative case study, where objects with $<100 \mu \mathrm{m}$ feature dimensions and elastic moduli spanning more than three orders of magnitude are demonstrated. Furthermore, good storage stability and network uniformity without thiol leaching makes the present method an appealing, simple approach to save both time and money in next generation $3 \mathrm{D}$ printing.

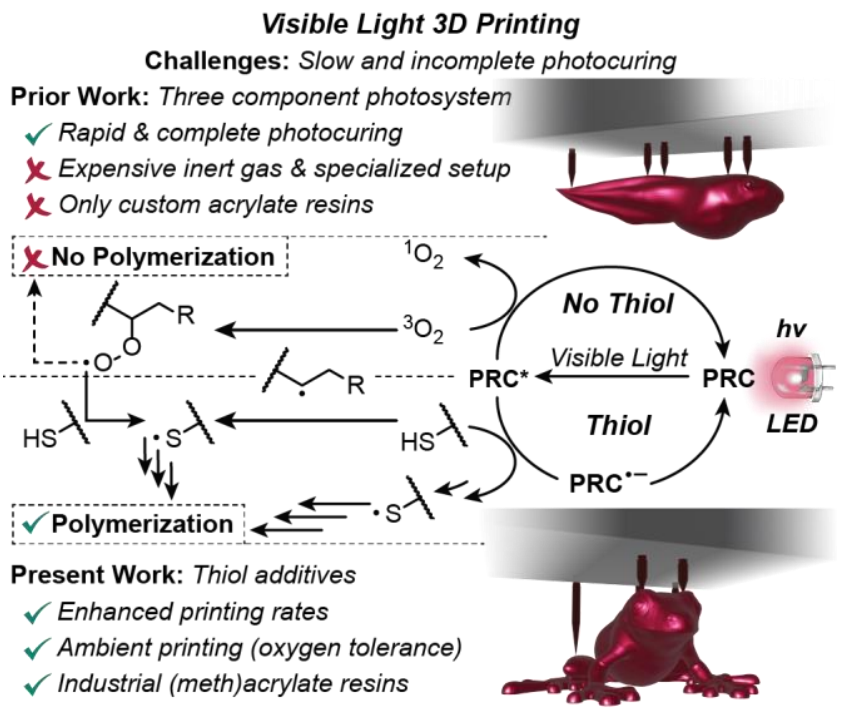

Figure 1. Challenges, prior work, and presented advancements in visible digital light processing (DLP) 3D printing. The central reaction mechanisms show how oxygen inhibits polymerization/curing (top, prior work) and how the addition of thiols bypasses inhibition to generate reactive thiyl radicals that induce polymerization/curing (bottom, present work). The digital 3D renditions of the tadpole and frog relate to prior and present work, respectively, as metaphors for a transformative advance that enables "operation" in air.

\section{Results and Discussion}

Rapid visible light 3D printing under ambient conditions required removal of oxygen to minimize inhibition periods. Initially, photocurable resins that undergo a thiol-ene mechanism were considered given the precedent for oxygen insensitivity enabled by rapid and efficient hydrogen atom abstraction from thiols to generate reactive thiyl radicals. ${ }^{[28]}$ For commercial 
relevance, it was desirable to use industrial (meth)acrylate monomers and crosslinkers as bulk resin constituents. The exemplary resin described below contains an oligourethane diacrylate from Dymax ${ }^{\circledR}(B R-741)$ together with tri(propylene glycol) diacrylate (TPGDA) in a 1:1 ratio, pentaerythritol tetrakis(3-mercaptopropionate) (PETMP) (1-10 wt $\%$, displacing TPGDA), a three component photosystem, 2,2,6,6-Tetramethyl-1-piperidinyloxy (TEMPO), and an opaquing agent (OA) (Figure 2a and Figure S1 in the SI). The versatility of this protocol was demonstrated using a wide range of monomers and crosslinkers supplementating those described above: Dymax ${ }^{\circledR}$ oligourethane-di(meth)acrylates (BR-345 and BR-952), N,Ndimethylacrylamide, trimethylolpropane triacrylate, isobornyl acrylate, and 2-hydroxyethyl (meth)acrylate (described later and in detail in the SI). Additionally, nine other small molecules were tested as possible oxygen scavengers, including various thiols, 1,3diphenylisobenzofuran, triphenylphosphine, and ascorbic acid which all underperformed relative to PETMP using equimolar amounts by functionality (details provides in the SI, Figures S8 and S9 and Table S9). For clarity, representative experiments are highlighted in the main text, which emphasize resins containing BR-741 and TPGDA.

The combination of BR-741 and TPGDA provides a robust hard plastic. The tetrathiol, PETMP, was selected in an attempt to maintain a similar crosslink density given that thiol-ene addition reactions occur through a step-growth mechanism in contrast to free radical acrylate polymerizations that operate by chain-growth (i.e., the functionality of a diacrylate can be equated to that of a tetrathiol) (Figure 2). The three component photosystem comprised zinc tetraphenylporphyrin (ZnTPP) as the red light absorbing photoredox catalyst (PRC), 2(butyryloxy)- $N, N, N$-trimethylethan-1-aminium butyltriphenylborate as the electron donor (D) (co-)initiator, and [4-(octyloxy)phenyl](phenyl)iodonium hexafluoroantimonate as the electron acceptor (A) (co-)initiator. This photosystem efficiently generates radicals that can (in the absence of oxygen) result in rapid curing $(<10 \mathrm{~s})$ of acrylate-based resins upon exposure to red light $(\sim 620 \mathrm{~nm}) .{ }^{[11]}$ A red light system was chosen, as opposed to green or blue, to emphasize 
the magnitude of the present results given that rapid photocuring with longer wavelengths of light is inherently more challenging due to the utility of lower energy photons. Inclusion of small amounts of TEMPO $(0.01 \mathrm{wt} \%)$ as a radical scavenger was found to effectively stabilize the resins and prevent unwanted background (or "dark") curing (Figures S2 and S3 and Table S4 in the SI). The OA selected was Sudan Black, a biological azo-stain ${ }^{[29]}$ that can passively absorb red light to induce cis/trans isomerization and limit the penetration depth of photons to cure only within the layer of interest. Thus, the presence of OA optimizes 3D printing resolution, particularly in the z-dimension (layer thickness), by preventing cure-through. As a control, bis-acylphosphine oxide (BAPO) was employed as a Type I photoinitiator (PI) operable under $405 \mathrm{~nm}$ (violet) light exposure, which replaced the three-component photosystem (Figure 2a).

The addition of PETMP was anticipated to reduce oxygen inhibition through two mechanisms: 1) hydrogen abstraction from thiols by peroxy radicals formed when propagating carbon centered radicals react with molecular oxygen ${ }^{[30]}$ (Figure 2b) and 2) reductive quenching of ZnTPP* (excited ZnTPP) by thiols ${ }^{[31]}$ (Figure 2c). While the first mechanism (peroxy radical quenching) has been the predominant rationalization for oxygen tolerance of thiol-ene reactions that are activated through a Type I UV/violet mechanism, we hypothesize that the second mechanism (reductive quenching) plays a more vital role in the present Type II visible light photosystem. This is attributed to long-lived, oxygen reactive PRC triplet states, which are needed to facilitate rapid curing with visible light. ${ }^{[22]}$ Thus, it is proposed that reductive quenching of $\mathrm{ZnTPP}^{*}$ (Figure 2c, cycle 1) competes with energy transfer to molecular oxygen $\left({ }^{3} \mathrm{O}_{2}\right)$ (Figure 2 c, cycle 2 ), which forms reactive singlet oxygen $\left({ }^{1} \mathrm{O}_{2}\right)$. Upon reductive quenching, thiol oxidation followed by hydrogen atom $\operatorname{transfer}^{[32]}$ results in the formation of thiyl radicals, which rapidly react with prevalent (meth)acrylate functionality through an anti-Markovnikov thiol-ene addition mechanism. Furthermore, the resultant sulfides (thioethers) may react with ${ }^{1} \mathrm{O}_{2}$ to form sulfoxides, ${ }^{[31]}$ precluding deleterious pathways (e.g., 
${ }^{1} \mathrm{O}_{2}$ reacting with the PRC) that could hamper curing. In this manner, the benefit of thiols for visible light photocuring (via a Type II mechanism) was proposed to have an unprecedented impact on minimizing the oxygen inhibition period and concomitant build times for emergent 3D printing applications.
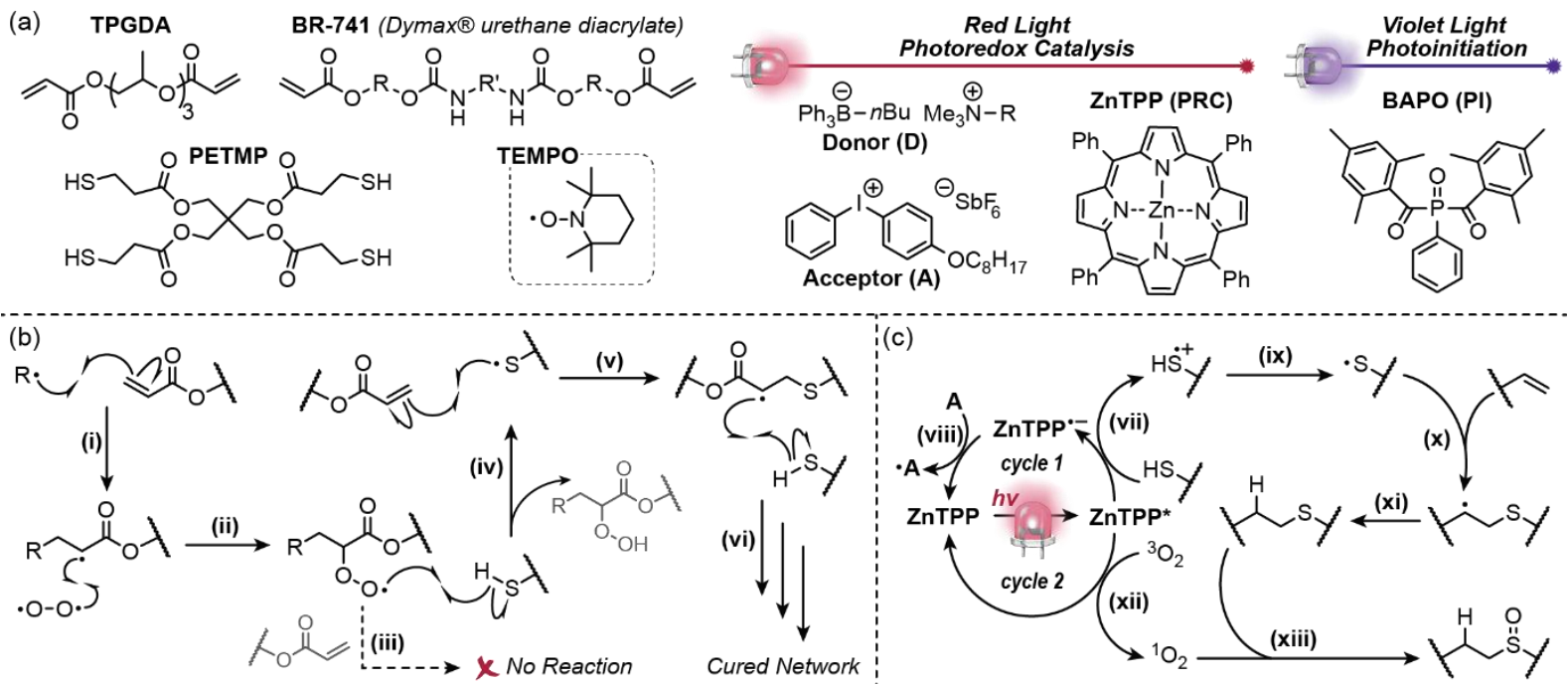

Figure 2. Primary resin components and proposed mechanisms that enable polymerization under ambient conditions. (a) Chemical structures for polymerizable groups. Red light reactive photosystem: ZnTPP (photoredox catalyst, PRC), borate derivative as electron donor (D) (co-)initiator, and diphenyliodonium derivative as electron acceptor (A) (co-)initiator. Violet light reactive photoinitiator (PI), BAPO. (b) Mechanism of radical addition to an acrylate (i) to generate a carbon centered radical that reacts with oxygen to form a peroxy radical (ii). The peroxy radical does not react with (meth)acrylates (iii), but abstracts a hydrogen from thiol to generate a thiyl radical (iv) that adds to (meth)acrylates via thiol-ene (v). Radical propagation results in polymer network formation (vi). (c) Cycle 1 mechanism: reductive quenching of photoexcited PRC (ZnTPP*) by thiols (vii) to generate $\mathrm{ZnTPP}^{--}$(radical anion) that can be oxidized back to ZnTPP by the diphenyliodonium acceptor, A, (viii) and a thiol radical cation that forms a thiyl radical upon $\mathrm{H}$-atom abstraction (ix). Thiol-ene addition (x), followed by $\mathrm{H}$ atom abstraction (xi). Cycle 2 mechanism: energy transfer from $\mathrm{ZnTPP} *$ to molecular oxygen $\left({ }^{3} \mathrm{O}_{2}\right.$ ) (xii) forming reactive singlet oxygen $\left({ }^{1} \mathrm{O}_{2}\right)$ that can oxidize sulfide to sulfoxide (xiii).

To determine the impact of thiol additives on the rate of ambient visible light photopolymerization, real time Fourier transform infrared spectroscopy (RT FTIR) was used to quantitatively determine monomer-to-polymer conversion during irradiation. Specifically, the disappearance of the signal corresponding to carbon-carbon double bond $(\mathrm{C}=\mathrm{C})$ stretches at $\sim 3,100 \mathrm{~cm}^{-1}$ was monitored using a transmission configuration with the resin sample residing in a $\sim 100 \mu \mathrm{m}$ gap between glass slides (additional experimental details found in the SI). ${ }^{[33]}$ All samples showed an excellent temporal response, with no identifiable reactions occurring in the absence of light during the first 10 seconds of each measurement. Initially, samples without 
PETMP (tetrathiol) were irradiated under ambient conditions with a red LED centered at 617 $\mathrm{nm}(\mathrm{FWHM} \approx 19 \mathrm{~nm})$ having an intensity of $2.5 \mathrm{~mW} / \mathrm{cm}^{2}$ (matching the DLP 3D printer intensity). The result was an inhibition period of $49.4 \pm 0.4 \mathrm{~s}$ and an initial rate of polymerization of $7.5 \pm 5.3 \% / \mathrm{s}$ (immediately following inhibition) (Figure 3a and Table 1). Degassing the same resin (no PETMP) with argon for $\sim 1$ minute resulted in a significant decrease in the inhibition period, down to $\sim 10 \mathrm{~s}$, analogous to prior observations. ${ }^{[11]}$ However, due to the high viscosity of the Dymax ${ }^{\circledR}$ BR-741 containing resin removal of oxygen by sparging with an inert gas was more challenging than our previous system, ${ }^{[11]}$ resulting in less reproducible inhibition periods. Furthermore, the presence of TEMPO supplements the inhibition period, particularly in the absence of an oxygen scavenger such as thiol (Figure S6 in the SI). The addition of only $1 \mathrm{wt} \%$ PETMP reduced the inhibition period to a greater extent than what was typically observed for degassed samples, down to $5.8 \pm 1.7$ s, while also increasing the polymerization rate up to $19.6 \pm 3.5 \% / \mathrm{s}$. Photorheology at equivalent light intensity under ambient conditions was in-line with these results, showing a time to gelation (gel point) of $7.9 \pm 0.8$ s post-irradiation with $1 \mathrm{wt} \%$ PETMP in contrast to a gel point of 64.0 \pm 0.6 s without PETMP (Figures S12-S16 and Table S12 in the SI). Increasing the content of PETMP further decreased the inhibition time (and gel point) and increased the rate of polymerization, with a nearly instantaneous reaction occurring upon exposure to light when 10 wt $\%$ PETMP was incorporated; for reference $10 \mathrm{wt} \%$ PETMP in the present resin is $\sim 10 \mathrm{~mol} \%$ PETMP (details provided in the "NMR Spectroscopy" section in the SI). Preliminary shelf life tests were performed by leaving the resins at room temperature in the dark and testing the photopolymerization kinetics with RT FTIR over time. After four weeks, it was observed that the rate of polymerization and inhibition period remained nearly unchanged, indicating good shelf stability (Figure S11 and Table S11 in the SI).

In support of the hypothesis that reductive quenching plays a vital role in minimizing oxygen inhibition, the three-component red light photosystem was compared to the one 
component (BAPO) UV/violet photoinitiation, holding everything else constant (Figure 3b and

Table 1). Irradiating the BAPO-containing resin with violet light $\left(405 \mathrm{~nm}\right.$ and $3.3 \mathrm{~mW} / \mathrm{cm}^{2}$ to match the DLP printing intensity) resulted in an inhibition period of $5.2 \pm 0.1 \mathrm{~s}$ and $2.4 \pm 0.2 \mathrm{~s}$ for samples containing 0 and $1 \mathrm{wt} \%$ PETMP, respectively. Thus, thiol additives provide a general "drop-in" method to significantly decrease cure time of commercial Type I UV/violet light activated resins, and show an even greater impact on Type II visible light activated resins. Overall, inhibition can be essentially removed for red-light activated resins via addition of 10 wt\% PETMP, and with only $1 \mathrm{wt} \%$ PETMP the resins still cure in under 10 seconds.
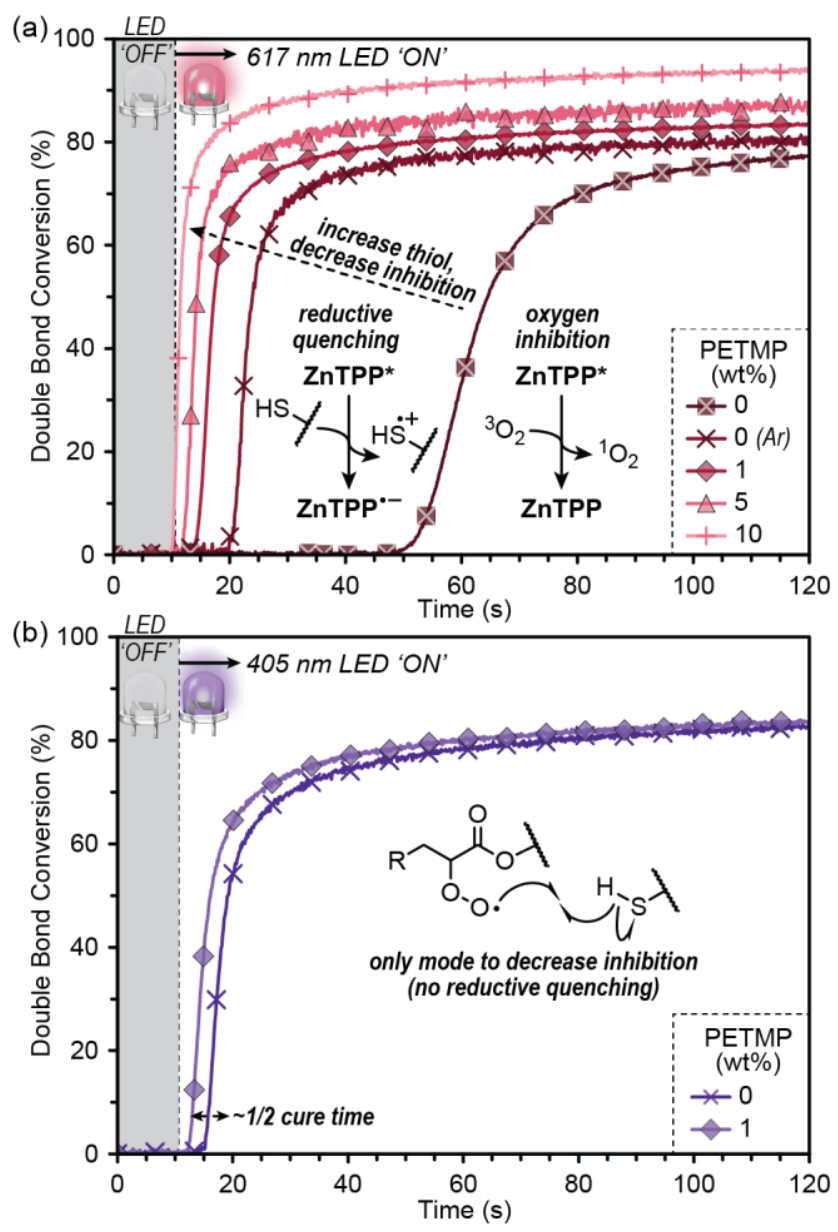

Figure 3. Influence of PETMP (tetrathiol) on oxygen inhibition and rate of BR-741:TPGDA (1:1) polymerization (where PETMP displaces TPGDA), monitored using real time Fourier transform infrared (RT FTIR) spectroscopy. (a) Red light photocuring $\left(617 \mathrm{~nm}, 2.5 \mathrm{~mW} / \mathrm{cm}^{2}\right)$ of resins without PETMP under an ambient (oxygen-containing) and inert (argon) atmosphere, and with 1, 5, and $10 \mathrm{wt} \%$ PETMP. (b) Violet light photocuring (405 nm, 3.3 $\mathrm{mW} / \mathrm{cm}^{2}$ ) using BAPO as the photoinitiator. A decrease in oxygen inhibition occurs with both red and violet light curing, but is more substantial for red light given the combination of reductive quenching and hydrogen atom abstraction by peroxy radicals. 
Table 1. Results from RT FTIR measurements shown in Figure 3, highlighting the impact of PETMP (tetrathiol) additives on both the oxygen inhibition period and apparent polymerization rate $\left(R_{p}\right)$.

\begin{tabular}{cccc}
\hline $\begin{array}{c}\text { PETMP } \\
{[\mathrm{w} \%]}\end{array}$ & $\begin{array}{c}\text { Irradiation } \\
\text { wavelength }(\mathrm{nm})^{\mathrm{b})}\end{array}$ & $\begin{array}{c}\text { Inhibition } \\
\text { Period }[\mathrm{s}]\end{array}$ & $\mathrm{R}_{\mathrm{p}}[\% / \mathrm{s}]^{\mathrm{c})}$ \\
\hline 0 & 617 & $49.4 \pm 0.4$ & $7.5 \pm 5.3$ \\
$0(\mathrm{Ar})^{\mathrm{a})}$ & 617 & $9.8 \pm 0.01$ & $16.6 \pm 1.4$ \\
1 & 617 & $5.8 \pm 1.7$ & $19.6 \pm 3.5$ \\
5 & 617 & $1.9 \pm 0.5$ & $25.4 \pm 2.3$ \\
10 & 617 & $1.0 \pm 0.4$ & $34.0 \pm 3.7$ \\
\hline 0 & 405 & $5.2 \pm 0.1$ & $13.0 \pm 1.0$ \\
1 & 405 & $2.4 \pm 0.2$ & $14.1 \pm 0.2$ \\
\hline
\end{tabular}

a)The resin was purged with argon for $\sim 1$ minute and the measurement was performed under an argon stream; ${ }^{\text {b) }} \mathrm{An}$ intensity of $2.5 \mathrm{~mW} / \mathrm{cm}^{2}$ for the $617 \mathrm{~nm}$ (red) LED and $3.3 \mathrm{~mW} / \mathrm{cm}^{2}$ for the $405 \mathrm{~nm}$ (violet) LED was used to match the intensity for digital light processing (DLP) 3D printing described later; ${ }^{\text {c) }}$ Rate determined using RT FTIR spectroscopy, monitoring the disappearance of the carbon-carbon double bond $(\mathrm{C}=\mathrm{C})$ stretch at $3100 \mathrm{~cm}^{-1}$ to determine the percent conversion of $\mathrm{C}=\mathrm{C}$ per second immediately following initiation (post inhibition period).

A critical parameter for additively manufactured objects is their mechanical performance, as this often dictates the application space. In particular, polysulfide thermosets from thiol-ene based resins often suffer from low stiffness and strength relative to (meth)acrylate-based resins owing to the flexibility of the C-S-C bonds and limited multifunctional thiol starting materials. ${ }^{[34,35]}$ As such, it was deemed important to characterize the impact of PETMP incorporation on mechanical properties, namely yield strength $\left(\boldsymbol{\sigma}_{y}\right)$, stiffness $(\boldsymbol{E}$, Young's modulus), and strain at fracture $\left(\varepsilon_{f}\right)$. Mechanical dogbone samples (ASTM D638 standard) were 3D printed with BR-741:TPGDA (1:1) resins containing 0, 1, 5, and $10 \mathrm{wt} \%$ PETMP (where PETMP displaces TPGDA), using red light $\left(\sim 620 \mathrm{~nm}, 2.5 \mathrm{~mW} / \mathrm{cm}^{2}\right)$ at an exposure time of 16, 14, 12, and $8 \mathrm{~s} /$ layer for resin containing 0, 1, 5, and $10 \mathrm{wt} \%$ PETMP, respectively, followed by uniaxial tensile testing (Figure 4a). Incorporating 1 wt\% PETMP into the resin resulted in samples with nearly identical (slightly improved) performance relative to those printed with no PETMP (under argon) $-\boldsymbol{\sigma}_{\boldsymbol{y}} \approx 30 \mathrm{MPa}$ and $\boldsymbol{E} \approx 1,000 \mathrm{MPa}$. Note, all freshly 3D printed (green) samples were post-cured with UV light for $\sim 20$ minutes to reach maximum conversion of vinyl functionality, as is standard practice. As the content of PETMP increased to 5 and $10 \mathrm{wt} \%$ a drop in $\boldsymbol{\sigma}_{\boldsymbol{y}}$ and $\boldsymbol{E}$ was observed - 25 and $900 \mathrm{MPa}(5 \mathrm{wt} \%)$ and 18 and $300 \mathrm{MPa}(10 \mathrm{wt} \%)$, 
respectively. Printing the samples with $1 \mathrm{wt} \%$ PETMP at three different edge-on angles, horizontal $\left(0^{\circ}\right)$, diagonal $\left(45^{\circ}\right)$, and vertical $\left(90^{\circ}\right)$ provided very similar ( isotropic) mechanical performance indicating good adhesion between the layers (Figure S28 and Table S17 in the SI). Thus, tailorable mechanical properties with multifunctional thiols was demonstrated, showing a strong and stiff plastic that matches the parent commercial acrylic resin at low thiol contents to a soft and ductile material with higher contents of PETMP, all while enabling rapid, low energy visible light 3D printing under ambient conditions.

To demonstrate the versatility of this platform, two other Dymax® resins capable of providing a stronger and more rigid (BR-952) or softer and more compliant (BR-345) material relative to BR-741 were examined (Figure 4b). Specifically, BR-952, a dimethacrylate, was mixed with hydroxyethyl methacrylate (HEMA) and PETMP in a 70/29/1 wt \% ratio, while BR345, a diacrylate, was mixed with hydroxyethyl acrylate and PETMP in a 50/49/1 wt\% ratio. In both cases, reproducibly rapid ambient photopolymerization with red light was demonstrated, revealing inhibition periods of $1.2 \pm 0.2 \mathrm{~s}$ and $1.3 \pm 0.2 \mathrm{~s}$ and $R_{p}$ of $6.0 \pm 0.3 \% / \mathrm{s}$ and $24.5 \pm$ $0.9 \% / s$ for BR-952 and BR-345, respectively (Figure S10 and Table S10 in the SI). Notably, this established for the first time that methacrylate-based resins could be 3D printed with visible light, albeit slower than analogous acrylate-based ones. Tensile testing of dogbones prepared from each resin provided distinct mechanical performance, from very strong and stiff $\left(\sigma_{\boldsymbol{y}} \approx 53\right.$ $\mathrm{MPa}$ and $\boldsymbol{E} \approx 1,400 \mathrm{MPa})$ for BR-952, to soft and extensible $(\boldsymbol{E} \approx 1.3 \mathrm{MPa}$ and $\varepsilon f \approx 80 \%)$ for BR-345. As an added bonus, the covalent incorporation of the thiols into the polymer network prevents unpleasant odors and leaching of potentially toxic components - as confirmed with gravimetric tests post solvent extraction (Figure S29 and Table S18) and dynamic mechanical analysis (Figure S30 and Table S19). These results indicate that addition of multifunctional thiols into commercially relevant acrylic resins represents a universal strategy to increase the rate of photocuring without compromising mechanical performance. 

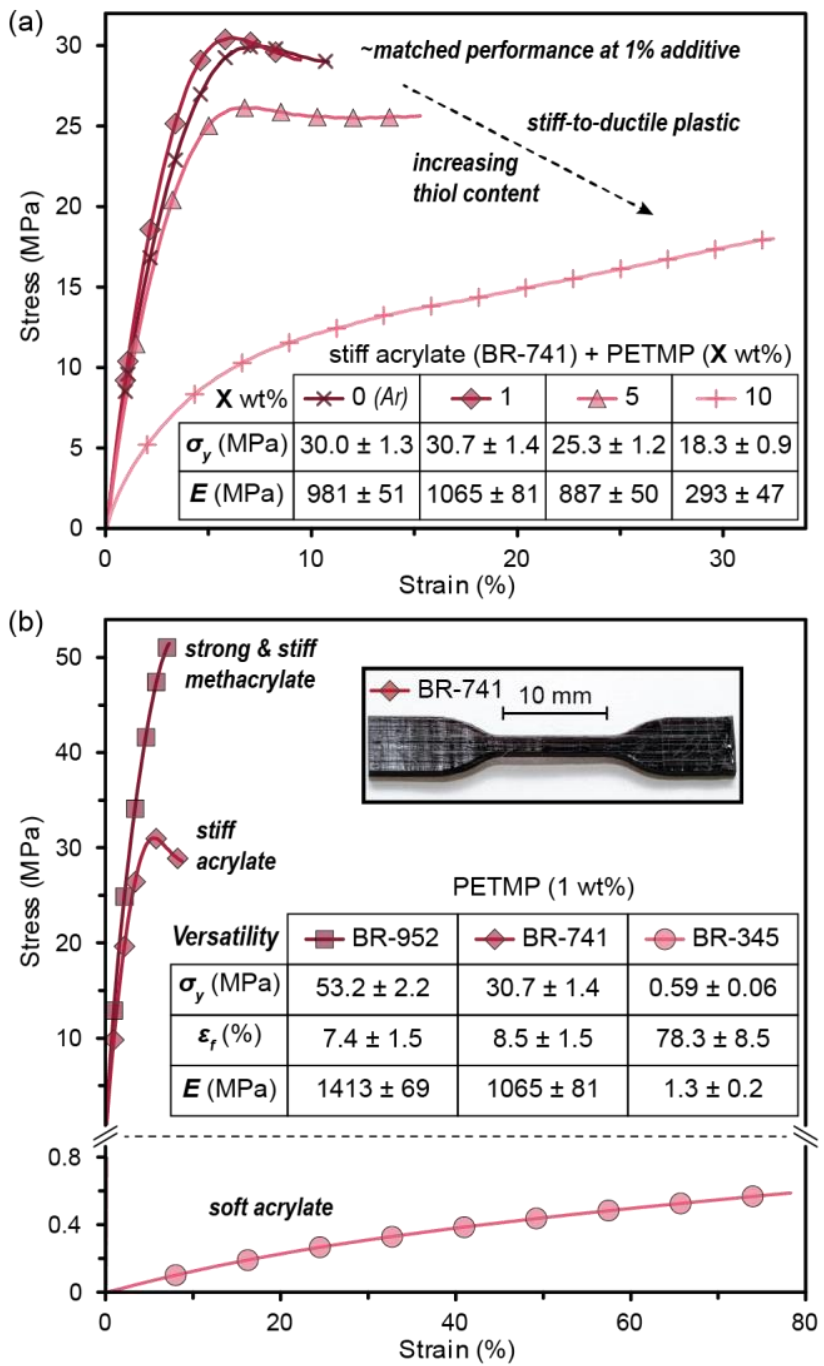

Figure 4. Stress-strain plots of red light 3D printed dogbones (ASTM D638 standard) under uniaxial tension. (a) Influence of PETMP (tetrathiol) content on yield strength $\left(\boldsymbol{\sigma}_{\boldsymbol{y}}\right)$ and stiffness (E, Young's modulus), showing that the high strength and rigidity can be maintained at low PETMP loading and that samples become more ductile at higher PETMP content. (b) Mechanical versatility from ambient visible light 3D printing with thiol additives, from three industrially relevant Dymax ${ }^{\circledR}$-containing resins. Insets show a representative digital image of the BR-741 dogbone and metrics for $\boldsymbol{\sigma}_{y}, \boldsymbol{E}$, and strain at fracture $(\varepsilon f)$.

Another important metric in additive manufacturing is feature resolution. To compete with commercial UV/violet-based DLP printing, our proposed system should be capable of providing features with $\leq 100 \mu \mathrm{m}$ lateral $(\mathrm{x}, \mathrm{y})$ and $25-100 \mu \mathrm{m}$ vertical $(\mathrm{z})$ resolution. To determine how thiol additives influence the minimum attainable feature size we used a custom digital method that gave control over exposure time within different locations of a single layer (details provided in the "Optical Profilometry" section in the SI). The method contained 12 different exposure times/layer, and within each time point a set of square pillars were present, with varying lateral dimensions from 1-16 volume elements (voxels) wide. The printer used in 
this study had a minimum voxel size of $20 \times 20 \mu \mathrm{m}^{2}$ in the lateral dimension and $25 \mu \mathrm{m}$ in the vertical dimension (i.e., layer thickness). The resolution as a function of PETMP content, number of layers (from 1 to 16), and exposure time/layer (from 2 to $24 \mathrm{~s} / 25 \mu \mathrm{m}$ layer at $2 \mathrm{~s}$ increments) was characterized using optical profilometry for BR-741:TPGDA resins cured with red light $\left(2.5 \mathrm{~mW} / \mathrm{cm}^{2}\right)$ (full digital images and additional optical profilometry images/analyses are provided in the SI, Figures S32-S42). The 3D topographical images of representative 12 s/layer time points are shown in Figure 5a and reveal the smallest visible square features as $\sim 160 \mu \mathrm{m}$-wide (8 voxels) for no PETMP (argon degassed), $\sim 80 \mu \mathrm{m}$-wide (4 voxels) for $1 \mathrm{wt} \%$ PETMP, and $\sim 20 \mu \mathrm{m}$-wide (single voxel) for 5 and $10 \mathrm{wt} \%$ PETMP. Impressively, surface area analysis of the $40 \mu \mathrm{m}$-wide squares ( $2 \times 2$ voxels) for the $5 \mathrm{wt} \%$ PETMP samples revealed an excellent match with the digital input file, while the surface area of $20 \mu \mathrm{m}$-wide squares was in experiment below theoretical values, indicating incomplete curing (Figure S39). Notably, the square features in the 1 and $5 \mathrm{wt} \%$ PETMP prints appear more well defined than those in the $10 \mathrm{wt} \%$ prints, which is attributed to a combination of the viscous resin getting trapped in the narrow $20 \mu \mathrm{m}$-wide cavities separating the squares and cure-through, particularly in the more reactive $10 \mathrm{wt} \%$ samples. This was corroborated by measuring the thickness and sidewall angle (SWA) at the corner of each time point to assess z-resolution (Figures S35-S38 in the SI). For all samples the thickness and SWA values nearly matched the digital file, measuring heights of $\sim 350-400 \mu \mathrm{m}$ (16 layers at $\sim 25 \mu \mathrm{m} /$ layer) and SWAs from $\sim 75-85^{\circ}$ (theoretical $=90^{\circ}$ ). Additionally, the maximum height was reached by 4 s/layer and 2 s/layer when incorporating $1 \mathrm{wt} \%$ PETMP and $10 \mathrm{wt} \%$ PETMP, respectively, and maintained until the longest time point generated at 24 s/layer. The stability in z-height over a wide range of exposure times/layer gave a large operating time window that enhanced reproducibility. This window is attributed to the combined absorption by the PRC (ZnTPP, $0.03 \mathrm{wt} \%$ ) and opaquing agent (OA, $0.002 \mathrm{wt} \%$ Sudan black) (Figures S43-45 in the SI). Altogether, resolution analysis of unoptimized 3D prints using the present ambient visible light method revealed that small features $(<100 \mu \mathrm{m})$ 
with high $\mathrm{x}, \mathrm{y}, \mathrm{z}$ fidelity (matching the digital input) were easily and reproducibly attainable, rivaling modern UV/violet-based DLP 3D printing technology. ${ }^{[1-3]}$

To underscore the capabilities of ambient visible light additive manufacturing, a complex hierarchical object was printed, specifically a 120-cell polytope (or dodecaplex), which is challenging or impossible to produce using traditional manufacturing processes (Figure $\mathbf{5 b}$ ). The optimized red light conditions were $14 \mathrm{~s} / 25 \mu \mathrm{m}$ layer, which correlates to a build rate of $\sim 6.4 \mathrm{~mm} / \mathrm{h}$. Impressively, the object contains 120 dodecahedral cells with the smallest feature size of $\sim 200 \mu \mathrm{m}$. Scanning electron microscopy (SEM) images show the individual $\sim 25 \mu \mathrm{m}$ layers from the additive process and emphasize the high feature fidelity. Images of additional complex 3D printed objects can be found in the supporting information (Figures S46-48). Uniquely, under UV excitation $(365 \mathrm{~nm})$ the objects emit red light, indicating that ZnTPP remains intact during the ambient printing, which provides an avenue towards high resolution 3D printing of "smart" functional plastics.
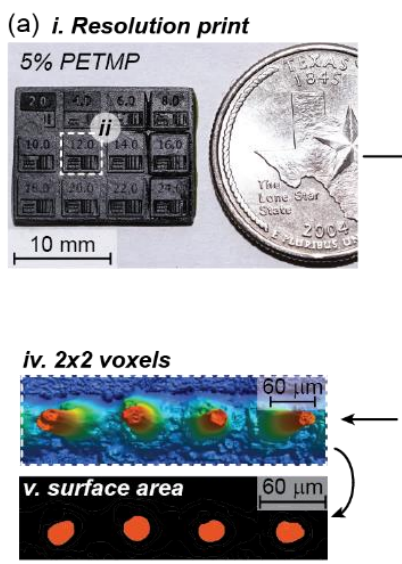

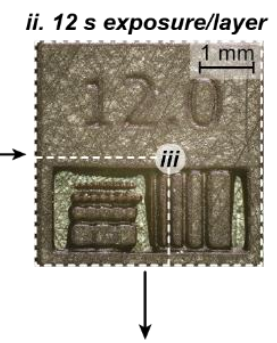

iii. 'Square' arrays
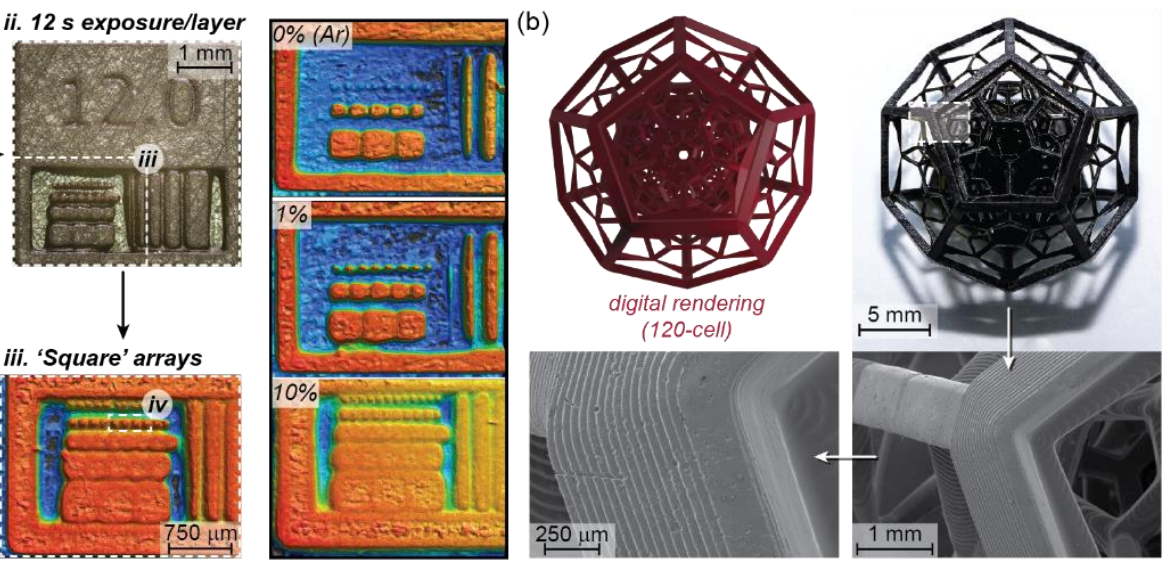

Figure 5. Resolution prints and 3D printed complex object using red light DLP with BR-741:TPGDA based resins (layer thickness $=25 \mu \mathrm{m}$ ). (a) Image of a representative resolution print with $5 \mathrm{wt} \%$ PETMP (left sequence) and analogous optical profilometry images for 0, 1, and $10 \mathrm{wt} \%$ PETMP prints (right). Starting from the top left for the representative sequence is (i) a digital photograph of the full print next to a quarter for reference, showing 12 different exposure times/layer, as indicated by the engraved numbers (representing seconds); (ii) Optical micrograph of the $12 \mathrm{~s}$ /layer section on the resolution print, which contains arrays of square pillars and lines; (iii) 3D topographic image showing square pillars with lateral dimensions varying from 1-16 voxels-wide (each voxel $=20 \times 20 \times 25 \mu^{3}$ ); (iv) Optical profilometry of 2 voxel-wide square pillars (lateral dimension) and corresponding image analysis showing good fidelity between digital input $\left(1600 \mu \mathrm{m}^{2}\right)$ and printed output $\left(2100 \pm 240 \mu \mathrm{m}^{2}\right)$. Overall, increasing PETMP content results in faster photocuring that provides access to smaller features, but at longer exposures can result in cure-through (loss in z-resolution). (b) Representative hierarchical 3D printed 120cell with the digital rendering (top left), photograph (top right) and SEM images (bottom). 


\section{Conclusion}

Energy efficient visible light 3D printing under mild ambient conditions was described. A tetrathiol additive (PETMP) as low as $1 \mathrm{wt} \%$ in commercially relevant acrylic resins was shown to enable rapid high resolution $3 \mathrm{D}$ printing with low intensity red light $\left(2.5 \mathrm{~mW} / \mathrm{cm}^{2}\right)$ in the presence of atmospheric oxygen. Monomer to polymer conversion was monitored using RT FTIR spectroscopy, revealing an excellent temporal response to light exposure in the presence of PETMP, with curing in less than 3 seconds. The dramatic reduction in oxygen inhibition time from $\sim 64 \mathrm{~s}$ to $\sim 8 \mathrm{~s}$ in adding $1 \mathrm{wt} \%$ PETMP, to essentially complete removal of oxygen sensitivity with $10 \mathrm{wt} \%$ PETMP, was attributed to a dual reaction of thiols to form thiyl radicals via hydrogen atom transfer to peroxy radicals and photooxidation by ZnTPP*. Furthermore, direct incorporation of PETMP into the acrylic network via a thiol-ene mechanism mitigates unfavorable odors and leaching of potentially toxic additives. The described method appears to be universal as it worked with a range of acrylates, acrylamides, and methacrylates, providing access to a diverse array of $3 \mathrm{D}$ objects with distinct chemical makeup and mechanical properties, from strong and stiff plastics ( $\boldsymbol{\sigma}_{\boldsymbol{y}} \approx 53 \mathrm{MPa}$ and $\boldsymbol{E} \approx 1,400 \mathrm{MPa}$ ) to soft and extensible elastomers $\left(\boldsymbol{E} \approx 1.3 \mathrm{MPa}\right.$ and $\left.\boldsymbol{\varepsilon}_{f} \approx 80 \%\right)$. Additionally, the inclusion of PETMP facilitated the formation of fine features with lateral dimensions $<50 \mu \mathrm{m}$ and a layer thickness of $25 \mu \mathrm{m}$, all while maintaining a wide processing window ( 6-24 s/layer) where little-to-no cure-through was observed. Finally, 3D printing of a hierarchical 120-cell polytope highlighted the capabilities offered by the present technique to harness custom objects with digitally defined form factors. This simple approach presents numerous opportunities to create next generation advanced materials in a mild additive fashion using visible-to-near infrared light, which will positively impact many arenas beyond 3D printing, from coatings and adhesives to imaging. Moreover, a number of fundamental scientific questions are now available to be examined, such as how various metrics (e.g., light dosage and wavelength) and resin compositions influence the speed, resolution, and material properties of thiol-containing visible light reactive resins. 
Currently, we are leveraging this new knowledge for 3D printing of hydrogels and multifunctional designer materials with applications in tissue engineering and soft robotics.

\section{Supporting Information}

Supporting Information is available.

\section{Acknowledgements}

We thank the Robert A. Welch Foundation (F-2007) and the Center for Dynamics and Control of Materials: an NSF MRSEC (DMR-1720595) for financial support. The authors acknowledge the use of shared research facilities supported in part by the Texas Materials Institute, the Center for Dynamics and Control of Materials: an NSF MRSEC (DMR-1720595), and the NSF National Nanotechnology Coordinated Infrastructure (ECCS-1542159). We thank Dr. Yun-Ho Jang at MonoPrinter for help with the custom DLP 3D printer and Dymax ${ }^{\circledR}$ for supplying a range of urethane (meth)acrylate oligomers for this study.

\section{References}

[1] R. L. Truby, J. A. Lewis, Nature 2016, 540, 371.

[2] A. Bagheri, J. Jin, ACS Appl. Polym. Mater. 2019, 1, 593.

[3] T. J. Wallin, J. Pikul, R. F. Shepherd, Nat. Rev. Mater. 2018, 3, 84.

[4] J. R. Tumbleston, D. Shirvanyants, N. Ermoshkin, R. Janusziewicz, A. R. Johnson, D. Kelly, K. Chen, R. Pinschmidt, J. P. Rolland, A. Ermoshkin, E. T. Samulski, J. M. DeSimone, Science. 2015, 347, 1349.

[5] M. P. De Beer, H. L. Van Der Laan, M. A. Cole, R. J. Whelan, M. A. Burns, T. F. Scott, Sci. Adv. 2019, 5, eaau8723.

[6] D. G. Moore, L. Barbera, K. Masania, A. R. Studart, Nat. Mater. 2019, DOI 10.1038/s41563-019-0525-y.

[7] J. J. Schwartz, A. J. Boydston, Nat. Commun. 2019, 10, 791.

[8] D. A. Walker, J. L. Hedrick, C. A. Mirkin, Science. 2019, 366, 360.

[9] S. K. Saha, D. Wang, V. H. Nguyen, Y. Chang, J. S. Oakdale, S. C. Chen, Science. 2019, $366,105$. 
[10] A. Vyatskikh, S. Delalande, A. Kudo, X. Zhang, C. M. Portela, J. R. Greer, Nat. Commun. 2018, 9, DOI 10.1038/s41467-018-03071-9.

[11] D. Ahn, L. M. Stevens, K. Zhou, Z. A. Page, ACS Cent. Sci. 2020, 6, 1555.

[12] A. Bagheri, C. W. A. Bainbridge, K. E. Engel, G. G. Qiao, J. Xu, C. Boyer, J. Jin, ACS Appl. Polym. Mater. 2020, $2,782$.

[13] C. A. J. M. Boyer, L. Zhang, X. Shi, Z. Zhang, R. P. Kuchel, R. Namivandi-Zangeneh, N. Corrigan, K. Jung, K. Liang, Angew. Chemie 2020, DOI 10.1002/ange.202014208.

[14] K. S. Lim, J. H. Galarraga, X. Cui, G. C. J. Lindberg, J. A. Burdick, T. B. F. Woodfield, Chem. Rev. 2020, acs. chemrev.9b00812.

[15] B. Strehmel, C. Schmitz, K. Cremanns, J. Göttert, Chem. - A Eur. J. 2019, 25, 12855.

[16] N. D. Dolinski, Z. A. Page, E. B. Callaway, F. Eisenreich, R. V. Garcia, R. Chavez, D. P. Bothman, S. Hecht, F. W. Zok, C. J. Hawker, Adv. Mater. 2018, 30, DOI 10.1002/adma.201800364.

[17] S. Bialas, L. Michalek, D. E. Marschner, T. Krappitz, M. Wegener, J. Blinco, E. Blasco, H. Frisch, C. Barner-Kowollik, Adv. Mater. 2019, 31, DOI 10.1002/adma.201807288.

[18] V. Hahn, P. Kiefer, T. Frenzel, J. Qu, E. Blasco, C. Barner-Kowollik, M. Wegener, Adv. Funct. Mater. 2020, 1907795.

[19] L. Michalek, S. Bialas, S. L. Walden, F. R. Bloesser, H. Frisch, C. Barner-Kowollik, Adv. Funct. Mater. 2020, 2005328, 1.

[20] N. S. Allen, J. Photochem. Photobiol. A Chem. 1996, 100, 101.

[21] P. Lu, K.-Y. Chung, A. Stafford, M. Kiker, K. Kafle, Z. A. Page, Polym. Chem. 2021, $12,327$.

[22] A. Stafford, D. Ahn, E. K. Raulerson, K. Y. Chung, K. Sun, D. M. Cadena, E. M. Forrister, S. R. Yost, S. T. Roberts, Z. A. Page, J. Am. Chem. Soc. 2020, 142, 14733.

[23] D. M. Arias-Rotondo, J. K. McCusker, Chem. Soc. Rev. 2016, 45, 5803.

[24] B. Husár, S. C. Ligon, H. Wutzel, H. Hoffmann, R. Liska, Prog. Org. Coatings 2014, 
77, 1789.

[25] S. C. Ligon, B. Husár, H. Wutzel, R. Holman, R. Liska, Chem. Rev. 2014, 114, 577.

[26] L. Chen, Q. Wu, G. Wei, R. Liu, Z. Li, J. Mater. Chem. C 2018, 6, 11561.

[27] C. C. Cook, E. J. Fong, J. J. Schwartz, D. H. Porcincula, A. C. Kaczmarek, J. S. Oakdale, B. D. Moran, K. M. Champley, C. M. Rackson, A. Muralidharan, R. R. McLeod, M. Shusteff, Adv. Mater. 2020, 32, 1.

[28] F. Dénès, M. Pichowicz, G. Povie, P. Renaud, Chem. Rev. 2014, 114, 2587.

[29] D. P. Penney, J. M. Powers, M. Frank, C. Willis, C. Churukian, Biotech. Histochem. 2002, 77, 237.

[30] C. E. Hoyle, T. Y. Lee, T. Roper, J. Polym. Sci. Part A Polym. Chem. 2004, 42, 5301.

[31] A. Guerrero-Corella, A. María Martinez-Gualda, F. Ahmadi, E. Ming, A. Fraile, J. Alemán, Chem. Commun. 2017, 53, 10463.

[32] L. Capaldo, D. Ravelli, European J. Org. Chem. 2017, 2017, 2056.

[33] S. Chatani, T. Gong, B. A. Earle, M. Podgorski, C. N. Bowman, ACS Macro Lett. 2014, 3,315 .

[34] H. B. Song, A. Baranek, B. T. Worrell, W. D. Cook, C. N. Bowman, Adv. Funct. Mater. 2018, $28,1$.

[35] M. Sahin, S. Ayalur-Karunakaran, J. Manhart, M. Wolfahrt, W. Kern, S. Schlögl, Adv. Eng. Mater. 2017, 19, 1. 